\title{
Implications of the Reanalysis and Weight of Evidence Determination of Human Health Studies for Exposure to Perchlorates under Cumulative and Aggregate Risk Assessment
}

\author{
Douglas Crawford-Brown'1,2, Sean Crawford-Brown ${ }^{2}$ \\ ${ }^{1}$ Cambridge Centre for Climate Change Mitigation Research (4CMR), Department of Land Economy, University of Cambridge, \\ Cambridge, UK \\ ${ }^{2}$ Cambridge Science and Policy Consulting, Cambridge, UK \\ Email:djc77@cam.ac.uk
}

How to cite this paper: Crawford-Brown, D. and Crawford-Brown, S. (2016) Implications of the Reanalysis and Weight of Evidence Determination of Human Health Studies for Exposure to Perchlorates under Cumulative and Aggregate Risk Assessment. Journal of Environmental Protection, 7, 1765-1790.

http://dx.doi.org/10.4236/jep.2016.712142

Received: October 19, 2016

Accepted: November 11, 2016

Published: November 14, 2016

Copyright $\odot 2016$ by authors and Scientific Research Publishing Inc. This work is licensed under the Creative Commons Attribution International License (CC BY 4.0).

http://creativecommons.org/licenses/by/4.0/

\begin{abstract}
This paper applies cumulative and aggregate risk methods and weight of evidence determination to re-analysis of epidemiological and clinical studies of exposure to perchlorates. The implications of cumulative and aggregate risk are considered for 28 epidemiological studies on IUI, serum thyroid hormone levels and clinical indicators. Consideration is given to simultaneous exposures to perchlorates, nitrates, thiocyanates and organohalogens in the study populations. The elevation of effects by perchlorates alone is found only in the studies that use urinary perchlorate as the metric of exposure. These studies are beset by a problem with use of urinary perchlorate concentration in that there is large inter-subject variability in the relationship between intake and urinary concentration due to differences in metabolism and disposition of the compounds following ingestion. As a result, an individual placed into the "high urinary concentration" group may be there due to high values of exposure, to long biological clearance half-lives, or due to high transfer fractions from the serum into the urine. The influence could be removed by correcting urinary levels by measured clearance half-times for individuals in a study, but that has not been done in the case of the studies examined here. It is of interest therefore that the studies that use direct measures of intake of perchlorates rather than urine concentration fail to display the hormone effects. The current study uses a "weight of evidence" approach for perchlorates, employing all 28 studies. The result is a slope of the exposureresponse curve (percentage change in hormone effect per unit exposure) of $0.3 \%$ per $\mu \mathrm{g} / \mathrm{kg}$-day, with $95 \%$ confidence interval of $(-0.05 \%, 1 \%)$. This confidence interval for the slope encompasses 0 , indicating no statistically significant slope when all data
\end{abstract}


are combined in a weight of evidence determination. This is consistent with the conclusions of the USEPA and EFSA that the epidemiological studies do not provide compelling evidence for a causal association between exposures to perchlorates and either hormone effects or clinically adverse effects. The conclusions are 1) that current epidemiological results do not provide evidence of effects induced by perchlorates apart from the IUI effects, 2) that the same results provide evidence that the IUI effects induced at environmental levels of exposure are associated with down-stream adverse effects and 3) that effective risk management requires the cumulative and aggregate risk framework adopted here, suggesting a need for risk assessors to return to the original studies and provide separate estimates of exposure-response relationships for all four compounds or at the least to control for exposures to nitrates, thiocyanates and organohalogens.

\section{Keywords}

Risk Management, Perchlorate, Cumulative Risk, Public Health

\section{Introduction}

Establishment of regulatory limits on exposure to environmental risk agents has traditionally focused on one compound at a time. This may in part be justified from a policy perspective, as it both simplifies the regulatory process and leads to regulatory limits that are applied equally in all circumstances. However, the science of human health risk assessment has evolved due to increasing recognition that risk agents interact at a variety of biochemical, cellular and physiological levels, with common modes and mechanisms of action [1] [2].

This has led to increasing calls for-and in some cases use of-more complex approaches to risk management based on aggregate and cumulative risk assessment [3]-[9]. Here, aggregate risk assessment refers to the same compound acting through different pathways of exposure, while cumulative risk refers to the combined effects of different compounds with the same mechanism of action.

While scientific understanding of cumulative and aggregate risk has advanced, they have not been applied fully in human health risk assessment underlying environmental regulations. Exceptions have been in the cases of radionuclides [10], dioxin-like compounds using a Toxicity Equivalence Factor [11] and mycotoxins [12] [13]. Each of these cases employs the concept of equivalency, either of intake or delivered tissue dose, to assess the combined actions of compounds falling within these categories.

A significant issue that arises in cumulative and aggregate risk is the point within regulatory risk assessment and management at which these ideas are applied. The earliest examples of their application took place following risk assessment, at the risk management stage. An example is the use of a Hazard Index (HI) and/or Relative Source Contribution (RSC) in the US. In both instances, risk coefficients for each individual compound were developed from epidemiological, clinical (human volunteer) and tox- 
icological studies in which incremental elevation of effects were attributed solely to incremental exposure to a single compound of interest, albeit perhaps with control for confounding by other compounds acting through the same mechanism of action, or otherwise known to influence the risk coefficient by modifying sensitivity.

However, these approaches to recognising the influence of aggregate and cumulative risk have generally not led to reinterpretation of the original data on which risk coefficients are based, reflecting the interactions of the compounds in generating these data. There are two broad approaches to incorporate cumulative and aggregate risk into the interpretation of primary data:

- Control for confounding where compounds other than the one of interest may also be present and acting through the same mechanism of action

- Reinterpretation of the original data to directly calculate equivalency-based risk coefficients, attributing the incremental elevation of adverse or potentially adverse effects to exposure to the mixture of compounds rather than to any single compound in that mixture.

By way of example, consider the effects of perchlorates on thyroid function. Regulatory risk assessments of perchlorates in both the US and EU have been based primarily on interpretation of the data from Greer et al. [14]. In that study, individuals were administered perchlorates in water over an extended period and the influence on Iodide Uptake Inhibition (IUI) assessed. Those data are shown in Figure 1.

The threshold value of ADRI associated with decline is approximately $0.01 \mathrm{mg} / \mathrm{kg}$-day or $10 \mu \mathrm{g} / \mathrm{kg}$-day, with thresholds of between $10 \mu \mathrm{g} / \mathrm{kg}$-day and $100 \mu \mathrm{g} / \mathrm{kg}$-day depending on the degree of IUI required for downstream adverse effects and the uncertainty factor chosen to establish a regulatory limit.

Interpretation of the Greer et al. [14] data in this manner has led to proposed regulatory limits in water (primarily in the US) and food (primarily in the EU). It is well established, however, that other compounds, principally including nitrates and thiocyanates, act through the same Sodium-Iodide Symporter (NIS) mechanism to produce IUI. An alternative approach is to reinterpret the Greer et al. [14] data through the lens
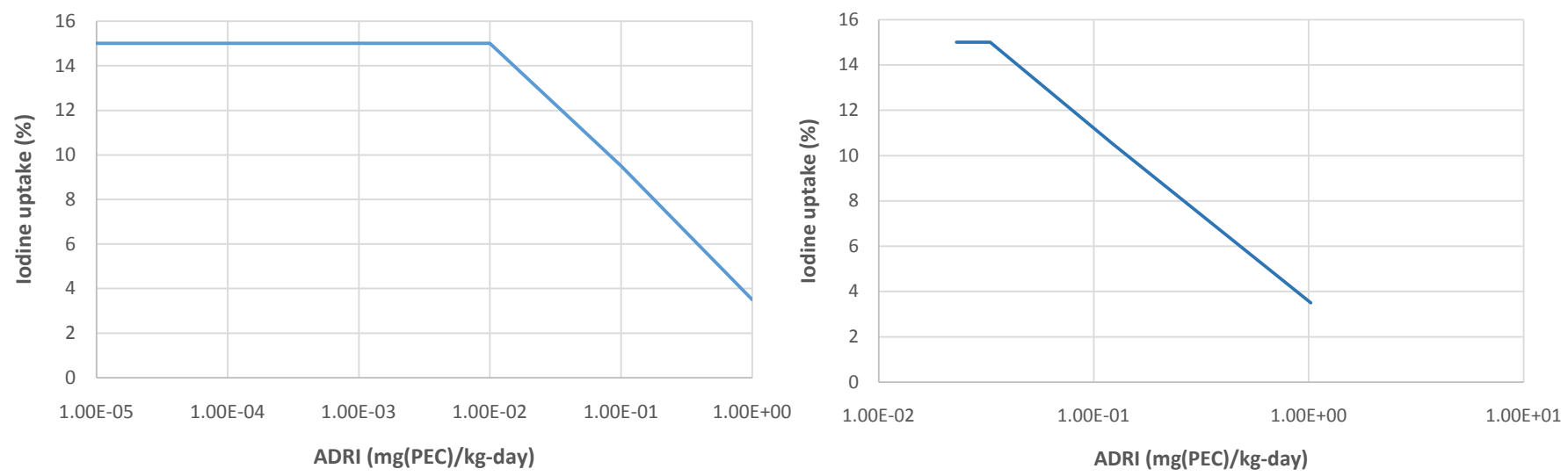

Figure 1. The original data of Greer et al. [14] focusing solely on the average daily rate of intake (ADRI) of perchlorates as the causative factor in the decline of iodine uptake in the thyroid. The left figure uses the units of perchlorate intake, while the right figure uses the units of PEC intake (as discussed below). 
of cumulative and aggregate risk, developing regulatory limits based on the concept of Perchlorate Equivalent Concentration or PEC [15]. Numerical values of PEC for perchlorates, nitrates and thiocyanates have been developed [16] [17] and applied in reinterpretation of the Greer et al. [14] primary data [18] [19]. Returning to Figure 1, the values of ADRI across the $\mathrm{x}$-axis would increase by approximately $24 \mu \mathrm{g}(\mathrm{PEC}) / \mathrm{kg}$-day due to background exposure to nitrates and thiocyanates.

While this reinterpretation of primary data from Greer et al. [14] through the lens of cumulative and aggregate risk has been carried out for perchlorates and their effect on IUI [18] [19], it has not been the norm for epidemiological studies. Instead, some of the studies have taken the first approach noted previously (control for confounding by nitrates and thiocyanates), although most have not applied even this limited procedure to account for the simultaneous actions of the three compounds. In addition, the studies do not control for exposure to organohalogens, a point which will become particularly significant in later sections when effects on concentration of serum thyroid hormone levels (and not simply IUI effects) are considered.

A related issue concerns the appearance-or lack of appearance-of effects from exposures to perchlorates at the regulatory limits being proposed based on the traditional approach to developing risk coefficients or thresholds for individual contaminants such as perchlorates. If such stringent regulatory limits are required by the action of perchlorates, why are they not evident in meta-analyses of the primary data? Several potential answers present themselves:

- The effects of perchlorates are masked by the actions of nitrates, thiocyanates and organohalogens when a study fails to control for confounding by these latter compounds, suggesting the need for such control for confounding.

- There is significant misclassification of exposures in the studies leading to bias towards the null, suggesting the need to improve exposure assessment and re-conducting the studies.

- The power of the individual studies is too weak to discern any such effects (hence the need to examine the multiple studies considered here).

- The effects of perchlorates at the low levels of regulatory limits proposed have been significantly over-stated through failure to account for cumulative and aggregate risk in interpreting the primary data of Greer et al. [14] using PEC.

This raises the primary research question of the current paper: Does consideration of cumulative and aggregate risk significantly alter the conclusions of those studies with respect to risks of exposure to perchlorates, and does the weight of evidence support claims that exposure to perchlorates at environmental levels are associated with changes in Iodide Uptake Inhibition, serum thyroid hormone levels and/or clinically adverse effects?

Before turning to this question, it is useful to review the summaries of epidemiological studies by the USEPA [20] and EFSA [21]. The USEPA [20] provides an analysis of epidemiological studies through 2012 focused on perchlorate exposures and alterations in thyroid function or circulating hormonal levels (primarily T3, T4, FT4, Tg or TSH) 
in adults, pregnant women and newborns. Results are summarised here, based on the conclusions in Table A-5 of the EPA report:

- Perchlorate was not associated with serum thyroid hormone levels in men.

- Effects in women were slightly increased TSH and decreased T4, but these effects were restricted to women at lower levels of urinary perchlorate.

- The effects in women were present even when control for thiocyanates was carried out.

- The effects were not seen in women with high levels of urinary perchlorate; this subpopulation also had control for exposure to nitrates in the blood.

- No increases in Tg or TSH and no decreases in FT4 among women in early pregnancy, late pregnancy, or neonates at birth related to perchlorate in drinking water were found. There were no differences in the metrics found across three cities with differing levels of perchlorate exposure. No relationship between perchlorate levels and neonatal thyroid function or fetal growth retardation was found.

- For first trimester women, there was no association between urinary perchlorate and serum TSH or FT(4) in the individual euthyroid or hypothyroid/hypothyroxinemic cohorts, even under multivariable regressions. (No analysis is performed for women past the first trimester).

- There was no association between urinary perchlorate concentrations and serum thyrotropin, free thyroxine index, or total triiodothyronine values. In multivariate analyses adjusting for urinary iodine concentrations, urinary creatinine, gestational age, and thyroperoxidase antibody status, urinary perchlorate was not a significant predictor of thyroid function.

- No change in neonatal T4 levels from maternal consumption of drinking water at levels similar to the MCL values under consideration by the USEPA was found.

- There is an association between perchlorate exposure and increased urinary TSH in infants with low urinary iodide (indicating iodine deficiency), consistent with previous findings. Higher exposures to thiocyanates and nitrates were also associated with higher TSH in these infants. Multiple regression was not performed so interactive effects cannot be determined.

Similarly, the USEPA's Science Advisory Board [22] concluded that “...epidemiologic studies published since the 2005 NRC report are insufficient to guide causal inference concerning an association between perchlorate exposure and thyroid dysfunction, or to support a derived MCLG".

With respect to the EFSA report [21], which extends the study period out to 2015, the relevant conclusions are summarised as:

- Administration of potassium perchlorate at levels of 200 to $900 \mathrm{mg} /$ day to individuals being treated have been "without any apparent complication".

- "Studies on healthy adult volunteers repeatedly exposed to 0.007 to $0.5 \mathrm{mg}$ perchlorate/kg b.w. per day for two weeks, as well as information from occupational studies, showed no correlation between the exposure to perchlorate and any adverse effects or significant changes in the thyroid hormone levels, even at exposure levels asso- 
ciated with a substantial inhibition of iodine thyroid uptake". This included levels of exposure associated with up to $67 \%$ IUI.

- "From the body of evidence available from medicinal use of potassium perchlorate, the studies on adult volunteers and the epidemiological studies in the occupational environment" show that "a sustained exposure to more than $0.4 \mathrm{mg}$ perchlorate ion/kg b.w. per day is likely necessary to cause hypothyroidism in normal adults, although a lower dose could be sufficient to cause adverse effects in pregnant women, infants, children and people with low iodine intake or pre-existing thyroid dysfunction". Note that this threshold is several orders of magnitude above that forming the basis of the more stringent proposed regulations. Even a reduction in the threshold by a factor of 10 for potential effects in pregnant women would produce regulatory limits on exposure much higher than the more stringent proposed limits.

- However, there is at least conflicting evidence of impacts on serum thyroid hormone levels in pregnant women and infants from retrospective epidemiological studies. The EFSA summarise these data as "...indicating changes in thyroid hormone levels in populations living in areas with known perchlorate contamination in drinking water $(>5 \mu \mathrm{g} / \mathrm{L})$. In particular, positive associations were often observed when potential exposure to perchlorate occurred in the presence of other risk factors, such as low dietary iodine intake, or exposure to other goitrogenic agents such as thiocyanate via tobacco smoke. The reported changes were of limited magnitude, were not associated with any disease and were not confirmed in other studies.

- "These epidemiological studies are of limited use for the evaluation of risks, because of the limited, or non-existent, information on exposure levels, the general natural high variability of the thyroid hormone levels, and the presence of possible confounding factors that are not taken into account in the analysis".

From the above two summaries [20] [21], it is evident that effects of exposures to perchlorates at environmental levels either are absent from epidemiological studies or are being masked by failure to include control for confounding by compounds such as nitrates and thiocyanates that also act by the NIS mechanism of action to influence IUI, or by compounds such as organohalogens [23] that are known to induce effects on serum thyroid hormone levels through endocrine disruption. The following analysis explores these possibilities to understand the apparent discrepancy between the more stringent proposed regulatory limits on exposures to perchlorates and lack of evidence for effects in epidemiological studies conducted at environmental levels of exposure.

\section{Epidemiological and Clinical Studies Database}

Systematic reviews of the epidemiological studies by Charnley [24] and Tarone et al. [25] show significant inconsistencies between the studies, resulting in meta-analyses with no statistical significance with respect to elevated effects when perchlorates alone are examined. Tarone et al. [25] attribute this partially to the effect of "...very low perchlorate exposure levels worldwide, compared with much higher levels of exposure to nitrate and thiocyanate, goitrogens that inhibit thyroid function by the identical me- 
chanism of action proposed for perchlorate".

If one considers down-stream effects in addition to IUI, similar conflicting findings are evident. A recent example is the study by Makey et al. [26] which examined the influence of exposure to organohalogens on thyroid function, controlling for exposures to perchlorates, thiocyanates and iodine. The organohalogens were associated solely with decreases in $\mathrm{TT}_{4}$ rather than other thyroid hormones, which the authors take as an indication that the compounds are acting to affect $\mathrm{TT}_{4}$ binding (a mechanism different to that of NIS effects and downstream of the IUI effects induced by perchlorates, nitrates and thiocyanates). No interaction effect was found between these three goitrogens and the organohalogen exposures.

It is especially significant that studies such as the one by Makey et al. [26] examining effects of exposure to organohalogens on thyroid hormone levels control for exposure to IUI goitrogens. However, the inverse has not been the case: control for confounding by organohalogens when examining the effects of exposure to the goitrogens. This is a significant omission given the evident ability of the organohalgens to induce changes in hormone levels.

The USEPA [20] provided an analysis of recent epidemiological studies focused on perchlorate exposures and thyroid function or circulating hormonal levels (primarily T3, T4, FT4, or TSH) in adults, pregnant women and newborns as of 2012. None of these studies examined more down-stream clinically adverse effects such as abnormal neurodevelopment, so they are indicative of potentially adverse effects rather than clinically adverse effects. Most include assessment of effects in pregnant women, women of reproductive age, women with iodine deficiencies, or smokers. Methods of exposure assessment varied from use of maternal residence (and associated perchlorate concentration in water) to urinary excretion data (see the earlier comment from the USEPA concerning the issue of poor exposure metrics in the epidemiological studies). Results are summarised here, based on the conclusions in Table A-5 of the EPA report.

The review by the EFSA [21] contains most of the studies cited by the USEPA, as well as studies published since 2012. They also include the study by Greer et al. [14] despite this being a clinical (human volunteer) study rather than an epidemiological study. However it is reviewed because it remains the primary study employed in both the US and EU for health effects of exposure to perchlorates in water. The studies considered here are those summarized by the EFSA [21] in Section 5.3.3 of their document).

Comparison of the epidemiological studies described below against that of Greer et al. [14] is complicated by the latter study not having the same dosing regime or length of exposure. The latter study is intermediate between a short-term and mid-term dosing regime. While the study extended for approximately 2 weeks, measurements of serum thyroid hormone levels were begun after the first day of dosing (although they also continue over the course of the study). The very high bolus doses administered raise the possibility that effects are attributable to the dose-rate, making it questionable to compare the results of Greer et al. [14] against the much longer term and more (environmentally) realistic dose-rates of the epidemiological studies. This issue is considered 
further in the Conclusions section.

The two sets of studies are combined below. The numbers assigned to each study are used in subsequent figures of this paper, both in this section and Section 3. For each study, the results as summarised by the authors are included, with no correction for cumulative or aggregate risk (this issue is explored in Section 3). In all studies, the results of chronic exposure only are considered, following the conclusions by both the USEPA and EFSA that acute exposures are not likely to be associated with effects. Only findings relevant in the current context of analysing the relationship between exposure to perchlorates and outcomes of IUI, serum thyroid hormone levels or clinically adverse effects are reported here.

1) Amitai et al. [27]. This epidemiological study assessed the relationship between exposure to perchlorate within mothers' drinking water during the gestation period and T4 levels in the blood. The highest exposure group (97 newborns) consumed water at up to $340 \mu \mathrm{g}$ perchlorate/L; the middle exposure group consumed water at $42-94 \mu \mathrm{g}$ perchlorate/L; the low exposure group consumed water with perchlorate concentration below $3 \mu \mathrm{g} / \mathrm{L}$. No statistically significant differences in T4 levels of the newborns were observed amongst the high and medium exposure groups compared with the control group.

2) Blount et al. [28]. 2299 (male and female, all $>12$ years of age) individuals were assessed for the relationship between urinary levels of perchlorate and thyroid functions measured by serum TSH and total T4 levels. There was control for confounding by age, sex, ethnicity, pregnancy status and body mass, but not for background exposures to nitrates and thiocyanates, nor for organohalogens. A correlation was found between perchlorate levels in urine and increased serum TSH levels, and an inverse correlation was found in women with low urinary iodine excretion between perchlorate levels in urine and serum total T4 levels. This latter finding was rejected upon subsequent analysis controlling for creatinine excretion. In subjects with higher (normal range) urinary iodine excretion, a correlation was found between perchlorate urinary levels and serum TSH levels, but not with serum T4 levels.

3) Braverman et al. [29]. 29 healthy adult workers were exposed through inhalation, at values of 10 to $300 \mu \mathrm{g} / \mathrm{kg}$-day of perchlorate. There was no control for confounding by background exposures to nitrates or thiocyanates, nor for organohalogens. IUI was decreased by $30 \%$ from the values found during the off-work period for the exposed workers, but no differences were found compared against a control population. No differences were observed in TSH and thyroglobulin levels. Small but statistically significant increases in total T3, total T4 and free T4 index were observed during the work shift compared against both the 3-day-off period and the control group. No effects on the thyroid were observed in the clinical examinations.

4) Braverman et al. [30]. 13 healthy adults were administered 0, 7 and $40 \mu \mathrm{g} / \mathrm{kg}$-day of perchlorate for 6 months. There was no control for confounding by background exposures to nitrates or thiocyanates, nor for organohalogens. No statistically significant IUI or thyroid functions changes were observed between the subjects in the three dosing groups. 
5) Brechner et al. [31]. This was an epidemiological study analysing the relationship between exposure to perchlorate in water and neonatal TSH levels. The exposed population consumed water at $6 \mu \mathrm{g} / \mathrm{L}$ or more for perchlorate (1099 newborns), while the control population (433 newborns) consumed water at levels at or below the Limit of Detection. There was control for age and ethnicity, but not for background exposures to nitrates or thiocyanates, nor for organohalogens. There was a statistically significant increase in mean TSH levels in the exposed compared against the control population.

6) Buffler et al. [32]. This was an epidemiological study analysing thyroid functions, with an exposed population consuming drinking water at above $5 \mu \mathrm{g} / \mathrm{L}$ for perchlorate and a control population consuming water at $5 \mu \mathrm{g} / \mathrm{L}$ or lower for perchlorate. There was no control for confounding by background exposures to nitrates or thiocyanates, nor for organohalogens. No association was found between exposure to perchlorate and cases of hypothyroidism.

7) Cao et al. [33]. This cross-sectional epidemiological study examined the relationship between exposure to perchlorate in water and urinary and serum TSH and T4 levels in 165 infants aged from $<48$ hours up to 12 months. Urine samples for the selected subgroup of 92 infants were also screened for concentrations of iodine, perchlorate, thiocyanate and nitrate. No statistically significant associations were found between urinary perchlorate levels and serum TSH and T4 levels.

8) Chang et al. [34]. This epidemiological study assessed the relationship between exposure to perchlorate in drinking water and indices of school performances and incidence of attention deficit hyperactivity disorder and autism in approximately 39,000 children. The control population was 8471 children from an area with perchlorate levels below the Limit of Detection, as well as with 7859 cases from the rest of state of Nevada. There was no control for confounding by background exposures to nitrates or thiocyanates, nor for organohalogens. No differences were observed between the exposed population and either control population in the measured indices of neurobehavioural disease or school performance.

9) Charatcharoenwitthaya et al. [35]. This epidemiological study in Thailand studied the effects of exposure to perchlorate and thiocyanate in 200 first trimester pregnant women aged 18 - 40 years (gestational age 14 weeks or less). Exposures were characterized as urinary perchlorate and thiocyanate levels. There was no control for nitrates or organohalogens. Effects measured were serum TSH, free T4 and free T3. Results included i) statistically significant positive correlations between perchlorate and thiocyanate levels and TSH levels and ii) statistically significant negative correlations between perchlorate and thiocyanate levels and free T4 levels and iii) a statistically significant positive correlation between perchlorate and TSH levels when perchlorate/iodine ratio is included in the analysis.

10) Crump et al. [36]. This epidemiological study examined the relationship between exposure to perchlorate in drinking water and indices of thyroid function in three villages in Chile. Exposures to perchlorate in the low exposure group were below the Limit of Detection; for the medium exposure group were $6.2 \mu \mathrm{g} / \mathrm{L}$ and for the high exposure 
group were $112 \mu \mathrm{g} / \mathrm{L}$. There was no control for confounding by background exposures to nitrates or thiocyanates, nor for organohalogens. Indices of circulating TSH and free T4 levels were obtained for 9784 newborns and 162 children aged six to eight years. No associations were observed between perchlorate exposure and the indices of thyroid functions or the presence of thyroid disease.

11) Greer et al. [14]. 24 healthy adults (12 male and 12 female) were administered 20, 100 or $500 \mu \mathrm{g} / \mathrm{kg}$-day of perchlorate for 14 days. In a second study 7 health adults ( 1 male and 6 female) were administered $7 \mu \mathrm{g} / \mathrm{kg}$-day of perchlorate. There was no control for confounding by background exposures to nitrates or thiocyanates. Results are shown in Figure 1 of the current paper.

12) Kelsh et al. [37]. This was an epidemiological study of the relationship between exposure to perchlorate in water and the incidence of primary congenital hypothyroidism in a population of 15,090 newborns in California. Exposures were in the range of $<4$ to $130 \mu \mathrm{g} / \mathrm{L}$. There was no control for confounding by background exposures to nitrates or thiocyanates, nor for organohalogens. The controls were 685,161 newborns from areas with groundwater perchlorate concentration below the Limit of Detection, as well with the overall California population. A lower number of cases were identified in the exposed population than in the non-exposed population. No statistically significant differences were observed for infant serum TSH levels in the exposed population compared against either control group.

13) Lamm et al. [38]. 37 healthy adult workers were exposed through inhalation, at values of $14,57,157$ or $486 \mu \mathrm{g} / \mathrm{kg}$-day of perchlorate. There was no control for confounding by background exposures to nitrates or thiocyanates, nor for organohalogens. No changes were found in serum total and free T4, total T3, thyroglobulin and TSH levels, thyroid hormone binding ratio and thyroid peroxidase antibodies, and no clinical abnormalities were noted in any exposure group.

14) Lamm and Doemland [39]. This was a case-control epidemiological study of the incidence of congenital hypothyroidism in 700,000 newborns in California and Nevada. Exposure was characterized as whether or not perchlorate was detected in drinking water, with a control group of the entire population of the states. No increased incidence in congenital hypothyroidism was observed. There was no control for confounding by background exposures to nitrates or thiocyanates, nor for organohalogens.

15) Lamm [40]. This was a follow-on epidemiological study for the Brechner et al. [31] study mentioned above, using similar methodology but different communities. It was carried out in part due to correct for lack of control for confounding with respect to elevations of the two cities in Brechner et al. [31]. Following introduction of this control for confounding, no statistically significant differences in TSH levels between exposed and control groups were observed. There was no control for confounding by background exposures to nitrates or thiocyanates, nor for organohalogens.

16) Lawrence et al. [41]. 9 healthy males were administered $140 \mu \mathrm{g} / \mathrm{kg}$-day of perchlorate for 14 days. There was no control for confounding by background exposures to nitrates or thiocyanates. Mean IUI at 14 days was $38 \%$, followed by a rebound of in- 
creased iodine uptake (higher than baseline by $25 \%$ ).

17) Lawrence et al. [42]. 8 healthy males were administered $40 \mu \mathrm{g} / \mathrm{kg}$-day of perchlorate for 14 days. There was no control for confounding by background exposures to nitrates or thiocyanates. Mean IUI at 14 days was $10 \%$, followed by a rebound of increased iodine uptake.

18) Leung et al. [43]. This epidemiological study examined the relationship between exposure to perchlorate and thiocyanate in breast milk and indices of infant serum thyroid functions in 64 breast-fed infants. The study excluded subjects with a history of thyroid disease, or who had undergone treatment with thyroid hormones, iodine-containing agents or contrast agents. There was no control for exposure to nitrates or organhalogens. No correlations were observed between perchlorate levels in breast milk or maternal urine and the index of thyroid function parameters in the infants.

19) Li et al. [44]. This was an epidemiological study of the relationship between perchlorate exposure in water and circulating thyroid hormonal levels in newborns in Las Vegas (17,000 newborns) and Reno (5882 newborns), Nevada. Two studies considered the comparison of newborns from two cities in Nevada, Las Vegas. Exposure was characterized by perchlorate levels in drinking water, with the water of Las Vegas displaying elevated levels compared against Reno (with perchlorate below the Limit of Detection). There was no control for confounding by background exposures to nitrates or thiocyanates, nor by organohalogens. Blood T4 levels were examined during the first four days after birth. No differences in circulating thyroid hormonal levels were found. A follow-up of 407 newborns (Las Vegas) and 133 newborns (Reno) similarly found no statistically significant differences in TSH levels between the two areas.

20) Li et al. [45]. This was an epidemiological study of the relationship between exposure to perchlorate in drinking water and incidence of thyroid diseases, including thyroid cancer. Sample population (exposed plus controls) was 177,141. There was no control for confounding by background exposures to nitrates or thiocyanates, nor by organohalogens. No increased incidence was observed in overall thyroid diseases or in any specific thyroid disease.

21) Mendez and Eftim [46]. 970 males and 907 females were examined using the NHANES database. "Pregnant women, individuals with thyroid diseases or with apparent hyperthyroidism, and subjects taking thyroid disease medications were excluded from the analysis". There was control for urinary excretion of bisphenol A and phthalate ester metabolites based on prior correlation of with thyroid hormone levels. There was no control for confounding by background exposures to nitrates or thiocyanates, or organohalogens. There was a statistically significant correlation between the increase in creatinine-adjusted urinary perchlorate levels and thyroid function parameters of decrease in total T4, free T4 and free T3 levels. Findings were consistent across male and female subjects, although simultaneous exposure to phthalates produced confounding.

22) Pearce et al. [47]. This cross-sectional epidemiological study examined the relationship between exposure to perchlorate in drinking water and indices thyroid functions in pregnant women. Examined effects included hypothyroid (high serum TSH 
levels) and/or hypothyroxinaemic (low serum free T4 levels) incidence, with follow-up including euthyroid women. There was no control for confounding by background exposures to nitrates or thiocyanates, or organohalogens. No statistically significant correlation was found between perchlorate intakes and indices of thyroid functions in any group, even for subjects with urinary iodine levels below $100 \mathrm{~g} / \mathrm{L}$ (taken to indicate potential iodine deficiency).

23) Pearce et al. [48]. This epidemiological study examined the relationship between urinary perchlorate levels in 241 first-trimester pregnant women and indices of thyroid function. There was no control for confounding by background exposures to nitrates or thiocyanates, or organohalogens. No association was found between urinary perchlorate concentrations and serum thyrotropin, free thyroxine index, or total triiodothyronine values, including within the subset of women with urinary iodine values less than $100 \mu \mathrm{g} / \mathrm{L}$. In multivariate analyses and adjusting for urinary iodine concentrations, no association was found between urinary perchlorate and urinary creatinine, gestational age, and thyroperoxidase antibody status.

24) Steinmaus et al. [49]. This study continues in the series using the NHANES data, assessing the impact of smoking and thiocyanate levels on the relationship between urinary per-chlorate and levels of serum thyroxine (T4) and thyroid-stimulating hormone (TSH). There was control for nitrates and thiocyanates serum levels in one of the subgroups. There was no control for background exposures to organohalogens. In women with urinary iodine levels less than $100 \mu \mathrm{g} / \mathrm{L}$, the association between perchlorate and decreased free $\mathrm{T} 4$ was greater in smokers than in nonsmokers. Results suggest that "thiocyanate in tobacco smoke and perchlorate interact in affecting thyroid function, and this effect can take place at commonly occurring perchlorate exposures. Agents other than tobacco smoke might cause similar interactions, and further research on these agents could help identify people who are particularly susceptible to perchlorate".

25) Steinmaus et al. [50]. This epidemiological study of 497,458 newborns examined the relationship between exposure to perchlorate in drinking water and odds ratios for high TSH levels. The higher exposure group consumed water above $5 \mu \mathrm{g} / \mathrm{L}$ of perchlorate, with the lower exposure group consuming water below $5 \mu \mathrm{g} / \mathrm{L}$ of perchlorate. Covariates of ethnicity, gender, age of newborns and of mothers, and feeding type (breast feeding, infant formula or both) were considered. There was no control for confounding by background exposures to nitrates or thiocyanates, or organohalogens. Odds ratios were calculated between the two exposure groups for the index of percentage with $\mathrm{TSH}>25 \mu \mathrm{U} / \mathrm{L}$ ("the cutoff concentrations at which neonates are screened for primary congenital hypothyroidism"); results were 1.53 and 0.72 when blood sampling was performed within the first 24 hours or after, respectively. Note the return to normal levels (or slight depression) after the first day. However, this return to normal levels after one day was not found in the newborns with the upper 95th percentile TSH levels.

26) Steinmaus et al. [51]. 1007 individuals were considered using the NHANES database. There was control for confounding by age, gender, ethnicity and urinary values 
for thiocyanates, but not for either nitrates or organohalogens. Median urinary perchlorate levels were $0.36-2.74 \mu \mathrm{g} / \mathrm{L}$ for the low urinary concentration group, $2.75-5.80$ $\mu \mathrm{g} / \mathrm{L}$ for the medium urinary concentration group and $5.82-47.80 \mu \mathrm{g} / \mathrm{L}$ for the high urinary concentration group. Urinary thiocyanate levels were $47-778 \mu \mathrm{g} / \mathrm{L}, 779-1900$ $\mu \mathrm{g} / \mathrm{L}$ and $1970-3200 \mu \mathrm{g} / \mathrm{L}$ for these three groups, respectively, indicating correlation between perchlorate and thiocyanate levels. There was an observed decrease by $3 \%-4 \%$ in total and free T4 levels in both the medium and high urinary level groups compared against the low urinary level group. No changes in TSH levels were observed in either group.

27) Suh et al. [52]. This study continues the series of studies based on the NHANES results, examining the relationship between exposure to perchlorate, nitrate and thiocyanate with changes in indices of thyroid hormone levels. Data from 1875 individuals were examined, including men with urinary iodine below $100 \mu \mathrm{g} / \mathrm{L}$, men with urinary iodine above $100 \mu \mathrm{g} / \mathrm{L}$, non-pregnant women with urinary iodine below $100 \mu \mathrm{g} / \mathrm{L}$, non-pregnant women with urinary iodine above $100 \mu \mathrm{g} / \mathrm{L}$, all pregnant women and pregnant women with urinary iodine below $150 \mu \mathrm{g} / \mathrm{L}$. A subset of 76 pregnant women was assessed using the later NHANES data. There was no control for organohalogens. Pregnant women with iodine levels less than $150 \mu \mathrm{g} / \mathrm{L}$ showed lower free T4 levels than the other groups. This group had the lowest creatinine-adjusted geometric mean concentration of urinary perchlorate, suggesting increased perchlorate levels (and presumably exposures) were correlated with higher free T4 levels in this group. No association was found between nitrate and thiocyanate urinary levels and indices of thyroid hormone levels for the groups. However, perchlorate and nitrate urinary concentrations were statistically significantly correlated with a decrease in free T4 levels for non-pregnant women only.

28) Tellez et al. [53]. This epidemiological study is a complement to study 10 above. It is a longitudinal epidemiological study in populations of Chile, assessing the relationship between exposure to perchlorate in water and indices of thyroid functions in mothers at early stages of gestation and in neonates at birth, as well as indices of growth retardations. Exposure of pregnant women to perchlorate through consumption of tap water was estimated to be $0.008 \mu \mathrm{g} / \mathrm{kg}$-day, $0.12 \mu \mathrm{g} / \mathrm{kg}$-day and $1.87 \mu \mathrm{g} / \mathrm{kg}$-day in the low, medium and high exposure groups, respectively. No differences were noted in examined indices of thyroid functions or neonatal growth parameters among the three exposure groups.

Figure 2 displays the quantitative results by study, again with no control or correction for cumulative or aggregate risk other than those applied by the authors of the original studies. In all instances, water concentrations are converted (where necessary) to Average Daily Rate of Intake (ADRI) by use of the age-specific body weights and water ingestion rates used in Crawford-Brown [18] for the perchlorate Monte Carlo analysis performed in that paper. For studies using urinary perchlorate as the exposure metric, the conversion to dietary intake rate uses the results of Leung et al. [54], English et al. [55] and Lau et al. [56], with a mean conversion factor of $0.015 \mu \mathrm{g} / \mathrm{kg}$-day per 


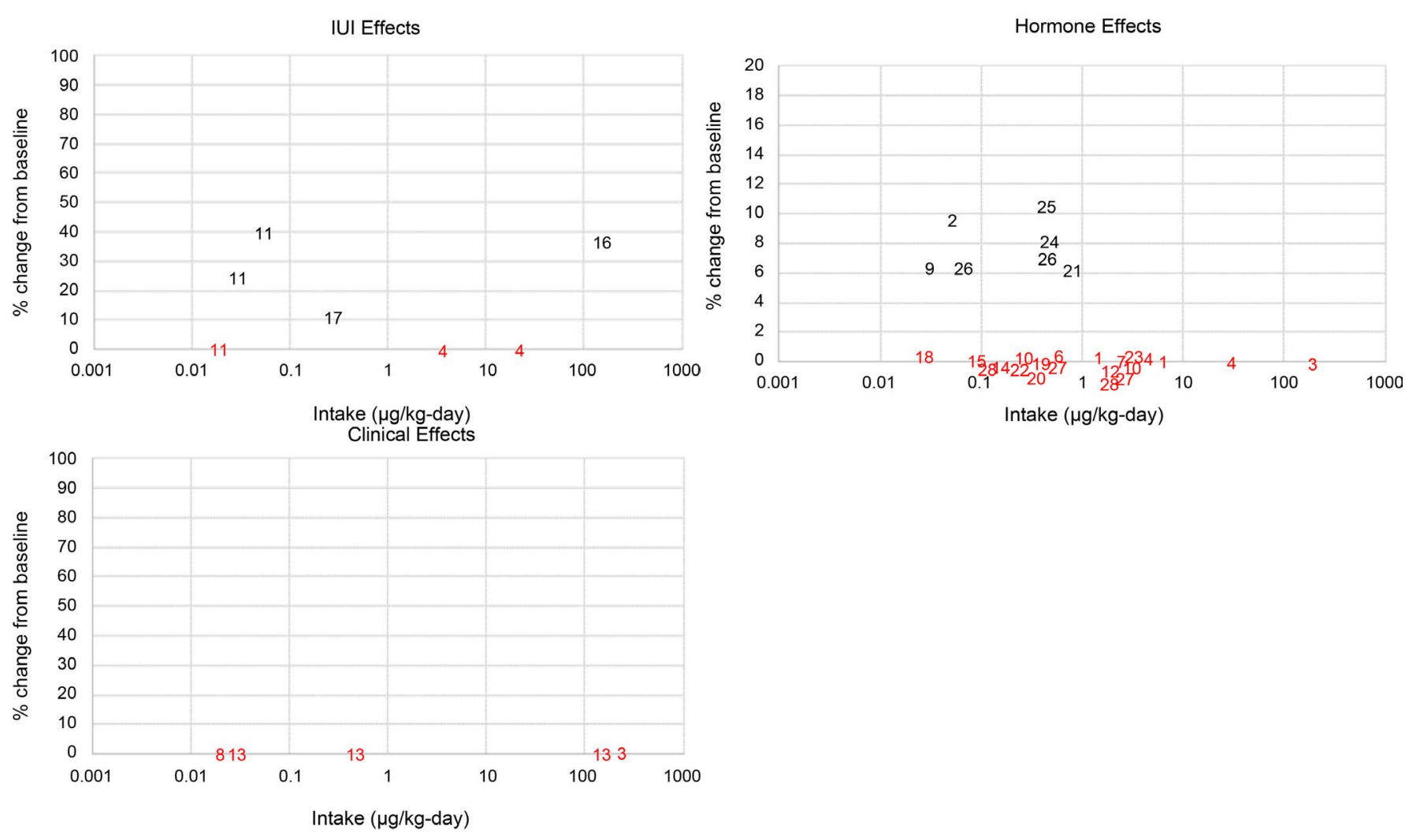

Figure 2. Summary of results from the 28 studies examined here, separated into the category of effect measured. Studies with no statistically significant change in effect are shown in red. In the figure for hormone effects, values below the $\mathrm{x}$-axis are all 0 (no statistically significant change). They are placed below the $\mathrm{x}$-axis simply for ease of locating them, as the exposure ranges for the separate studies overlap.

$\mu \mathrm{g} / \mathrm{L}$ (urine). These conversions allow comparison with the results of Greer et al. [14] summarised in Figure 1 by expressing all exposures in units of ADRI, although note should be taken of the differences between the dosing regime and timing of measurements of the Greer et al. [14] and the large majority of epidemiological studies (the latter being primarily chronic exposures at environmentally relevant dose-rates).

Where a study uses exposures categories, the central value of the exposure range is used here. Where there are only two categories characterised by a single value (for example, $<1 \mu \mathrm{g} / \mathrm{kg}$-day and $>5 \mu \mathrm{g} / \mathrm{kg}$-day), the former exposure category is set to the central value (for example, to $2.5 \mu \mathrm{g} / \mathrm{kg}$-day) and the latter exposure category is set to the median value of the exposures above this lower limit, assuming a lognormal distribution with the lower $95^{\text {th }}$ percentile at this low limit ( $5 \mu \mathrm{g} / \mathrm{kg}$-day) and Geometric Standard Deviation (GSD) of 1.5 as in Crawford-Brown [18].

In Figure 2, the effects have been divided into three categories: 1) IUI, 2) serum levels for thyroid-related hormones and 3) clinically adverse effects. Quantitative changes in any index of effect are always shown as percentage change compared against the baseline value reported in the study, whether representing an increase or decrease.

Two features of the above figures are especially notable. First, the IUI effects in the study by Greer et al. [14] are parallel to those of the other studies, but occur at significantly lower intakes. Second, the effects on serum thyroid hormone levels appear at the 
lower intakes of perchlorates but not at the higher intakes. These two points are reconsidered in the next section when cumulative and aggregate risk methods are applied.

\section{Implications of Cumulative and Aggregate Risk in Interpretation of Epidemiology Studies}

The analyses of Section 2 are re-considered here in light of cumulative and aggregate risk, following the arguments and exposure adjustments in Crawford-Brown [18]. A central part of the argument advanced here is that intakes of nitrates and thiocyanates are significantly larger-by more than an order of magnitude when expressed in units of PEC-than intakes of perchlorates in the epidemiological studies not involving human volunteers. When expressed in units of $\mu \mathrm{g}$ (PEC) $/ \mathrm{kg}$-day, the intakes of nitrates and thiocyanates are assumed to have combined values of $22.8 \mu \mathrm{g}(\mathrm{PEC}) / \mathrm{kg}$-day in these studies of environmental levels of exposure, consistent with the values assumed in Crawford-Brown [18].

This direct comparison of intakes of perchlorates, nitrates and thiocyanates is most applicable to the IUI effects, since these effects are produced primarily through changes in the NIS mechanism of action for all three compounds. The possibility remains that all three compounds may act by different, and dissimilar, mechanisms for the other two categories of effects-hormone effects and clinical effects. However, at least some contribution to these other two categories of effect will be through this shared NIS mechanism of action.

What can one conclude from the three components of Figure 2 when cumulative and aggregate risk are considered? Focus here will be on the effects on serum thyroid hormone levels, since the IUI effects have already been the basis of regulatory risk assessments, and the implications of cumulative and aggregate risk were explored in previous papers [18] [19]. There are no implications for the clinical effects component of Figure 1, since no statistically significant results were obtained for exposure to perchlorates at realistic environmental levels. The sole caveat to this statement is that exposures to nitrates, thiocyanates and organohalogens might cause a masking of the effects of perchlorates, although this would only be the case if there were differential exposures between the exposed and control groups, and if that difference led to the control group having higher background exposures to these three compounds than the "perchlorate exposed" group. There is no evidence that this is the case.

Where there is non-differential exposure (in the sense that exposure is unaffected by disease state), and where confounding is present due to failure to include control for exposure to compounds that act to produce the same effect, the result is usually "bias towards the null" [57]. This would mean that when developing risk estimates for exposure to perchlorates, failure to control for exposures to compounds such as nitrates, thiocyanates and organohalogens that produce the same effects on hormone levels would tend to have any of three effects: 1) the statistical significance of any finding of effects from perchlorates would be reduced, 2) the exposure-response slope or relative risk coefficient for perchlorates would be reduced and/or 3) the apparent threshold for 
effects would be increased. Each of these three possibilities suggest that in the case of confounding exposures that are non-differential with respect to exposure to perchlorates, the studies examined here would tend to understate the risk of perchlorates when there is no control for confounding by nitrates, thiocyanates and organohalogens (which is the case for most of the studies considered).

However, there is evidence that these confounding exposures are indeed correlated to some extent with that of perchlorates; this is especially the case for nitrates. The database of the EFSA demonstrates that those vegetables highest in perchlorates are also highest in nitrates, and green vegetables such as broccoli bio-concentrate perchlorates, nitrates and thiocyanates. This is in part to be expected given the common role of water in bioaccumulation of these vegetables by all three compounds. As a result, it is likely that individuals in high categories of exposure to perchlorates will similarly be in higher categories of exposure to at least nitrates and thiocyanates, since their exposures are controlled in large part by the relative intakes of green vegetables in the diet (and that intake is the same regardless of the compound considered).

Therefore, confounding exposures in the case of the epidemiological studies cited are unlikely to be non-differential with respect to the exposure categories for intakes of perchlorates. This is certainly the case for exposures to nitrates and thiocyanates; the implication is less clear for organohalogens. Since the assumption of non-differential exposure is not valid here (i.e. exposure to the confounders correlates with exposure to perchlorates), there is no expectation of bias towards the null. If anything, there would be "bias away from the null" when control for confounding exposures is not included. The impact of this on the studies examined here cannot be determined at present, as it would require re-conducting each study with control for exposures to nitrates, thiocyanates and organohalogens.

Focusing on the effects on serum thyroid hormone levels, note first that studies 3 and 4 are the only studies in which the intake of perchlorate was significantly higher than for the other compounds expressed in units of PEC. These studies therefore reflect the case where exposure to perchlorates are the dominant cause of any effects, if such effects are present, with little influence of cumulative and aggregate risk. And yet in each of these studies, there is no statistically significant change in hormone levels following exposure, despite these exposures being several orders of magnitude higher than the studies that do show an effect on hormone levels.

Several of the studies contained subsets of individuals for which confounding by nitrates and thiocyanates could be assessed. In the instance of study 7 , the apparent effect of exposure to perchlorates without control for confounding by the other IUIs was reversed when individuals exposed to perchlorates were sub-categorised by urinary levels of nitrates and thiocyanates. When this form of cumulative risk methodology was applied, there was no statistically significant elevation for perchlorate.

Focusing next on studies 21, 24 and 26, all show statistically significant alteration of serum thyroid hormone levels at much lower intakes of perchlorates. These studies were conducted using indirect metrics of exposure, namely urinary levels of perchlorate. 
The one study to control for confounding by the other IUIs (although not for organohalogens) was study 24. In that instance, when control for urinary levels of nitrates and thiocyanates was carried out, the error bars on indirect markers of hormone effects (the category of effects for which control for confounding was included) encompassed 0 . In addition, the effect of perchlorate was significantly altered by the presence or absence of smoking, urinary cotinine or urinary thiocyanates.

Study 26 shows strong correlation between levels of urinary perchlorates and thiocyanates. While perchlorates were associated with changes in free T4 in serum, they were not associated with changes in TSH serum levels (the reverse of the finding in study 2 once correction for creatinine excretion was applied). Given the significance of the correlation between urinary perchlorates and thiocyanates, and the much lower perchlorates levels than thiocyanates, small amounts of exposure misclassification for thiocyanates could significantly affect the perchlorates analysis of the relationship to free T4.

This correlation between serum/urinary perchlorates and thiocyanates has implications for study 25 , which was the sole study that showed a statistically significant relationship between exposure to perchlorates in water (characterized by the direct measure of water concentration rather than indirect measures of serum or urinary levels) and serum hormones (TSH), since there was no control for confounding by nitrates, thiocyanates and organohalogens. Given the difference in biological clearance half-times from the body for thiocyanates relative to perchlorates [17], failure to account for at least exposure to thiocyanates in the diet and smoking in study 25 could lead to spurious correlations between exposure to perchlorates and effects on serum thyroid hormone levels. The same applies to all other studies without such control for confounding, and extends to failure to include confounding by exposure to nitrates in food and water.

The studies based on urinary perchlorate as the measure of exposure suffer from an important problem of misclassification of exposure. Consider that regulatory limits focus on environmental concentration of a compound $(\mu \mathrm{g} / \mathrm{L})$, with the concentration limit based on a tolerated daily intake ( $\mu \mathrm{g} / \mathrm{kg}$-day). Epidemiological studies should therefore have as the measure of exposure either environmental concentration or daily intake, or at least a surrogate measure that is highly correlated with one of these two on an individual (not merely group mean) basis.

The relationship between urinary concentration and daily intake in an individual however is a function of both the rate at which this intake enters the transfer compartment of the body (primarily serum, but with some uptake into body water) and the first order rate constant for removal from this compartment to that of the urine (or bladder). It is also a function of diluting volume, although this can be corrected for through use of creatinine excretion. Let IR be the rate of intake into the body and $\lambda$ be the first order rate constant. The amount of the compound in the urine, $\mathrm{N}(\mathrm{t})$, is then proportional to $(\operatorname{IR} / \lambda)^{*}(1-\exp (-\lambda \mathrm{t}))$. At equilibrium, this content equals $(\operatorname{IR} / \lambda)$.

Using the results of Steinmaus et al. [51] as an example of the issue being raised, note 
that the three exposure groups are characterized by median urinary perchlorate concentrations of approximately $2 \mu \mathrm{g} / \mathrm{L}, 5 \mu \mathrm{g} / \mathrm{L}$ and $10 \mu \mathrm{g} / \mathrm{L}$. In that study (as in the others that use urinary perchlorate as the measure of exposure), it is assumed that the ratios of these three levels of urinary perchlorate are also measures of the ratios of environmental concentration or intake. However, biological rate constants vary by a factor of 3 or more between individuals. Therefore, two individuals with exactly the same value of IR may have differences in urinary concentration of a similar factor of 3 or more. This raises the significant possibility that the 'exposure categories' in this study (and the others using urinary perchlorate) are not in fact categories related to exposure (IR), but rather categories related to inter-subject differences in the first order rate constant $(\lambda)$. Individuals in the higher "exposure categories" of those studies are there due to some combination of higher value of IR and lower value of $\lambda$. In fact, the inter-subject variability in $\lambda$ could by this argument almost entirely account for the differences in urinary perchlorate in the three exposure groups. One is looking therefore not at "exposure categories" but rather at " $\lambda$ categories", with higher effects in the higher "exposure groups" arising because a low value of $\lambda$ in an individual provides greater time for the perchlorate to exert any effect it might have on the measured serum thyroid hormone levels.

This explanation would also account for the finding in Steinmaus et al. [51] that urinary perchlorate and urinary thiocyanate are fully correlated. The low "exposure category" individuals had the lowest perchlorate and thiocyanate concentrations, with the same pattern holding for the middle and high "exposure categories". Significantly, the ratio of urinary thiocyanate in the middle/low and high/low categories is the same factor of 3 to 4 one finds for urinary perchlorate. If the three categories considered "exposure categories" in that study are in fact " $\lambda$ categories", reflecting inter-subject variability in clearance rather than exposure (IR), this complete correlation between urinary perchlorate and thiocyanate would be explained. The same holds by implication for the other studies using urinary values rather than actual values of exposure.

The above argument is not that urinary concentration is unrelated to level of exposure. On average, people with higher level of exposure will have higher urinary concentration. However, the epidemiological studies cited suffer from the inverse of this issue by assuming that people with higher urinary concentration therefore have higher exposure, and then using this assumption to classify study subjects into exposure categories. This inverse relationship does not however hold, and so there is a significant possibility of exposure misclassification in interpreting those studies as indicative of an exposure-response relationship.

\section{Conclusions}

Consider the research question posed in Section 1: Does consideration of cumulative and aggregate risk significantly alter the conclusions of those studies with respect to risks of exposure to perchlorates, and does the weight of evidence support claims that exposure to perchlorates at environmental levels are associated with changes in Iodide 
Uptake Inhibition, serum thyroid hormone levels andl or clinically adverse effects?

Regulatory limits on exposure to compounds have traditionally been based on identifying a single "critical study", and using that study as the basis for quantitative estimates of risk coefficients or thresholds. Perchlorate is a strong example of this given the dominance of the Greer et al. [14] results in regulatory assessments. If a more robust "weight of evidence" approach is used, the full body of studies of Figure 2 would be used to establish a regulatory limit.

However, both the USEPA [20] and EFSA [21] (in the reports they cite) conclude that the epidemiological studies of hormone effects and clinical effects of Figure 2 are insufficient to establish either causality or a numerical estimate of the threshold. This conclusion follows primarily from the arguments advanced above: 1) that the results are inconsistent between the studies, 2) that there is inadequate control for confounding by compounds known to cause changes in thyroid hormone levels (including organohalogens) and 3) that exposure measures are poorly established or indirect. In a "weight of evidence" determination, a meta-analysis is performed of the studies to determine whether the collective result from the combined studies suggests a causal connection (where only a test for significance is conducted) or provide a numerical value of the risk coefficient (in Figure 2, the risk coefficient would be percentage change in the effects measure per unit exposure).

Using all of the studies and weighting by the standard error of the mean for each study, the slope of the exposure-response curve (percentage change in hormone effect per unit exposure) is $0.3 \%$ per $\mu \mathrm{g} / \mathrm{kg}$-day, with $95 \%$ confidence interval of $(-0.05 \%$, $1 \%)$. Results are shown in Figure 3. Note the confidence interval for the slope encompasses 0 , indicating no statistically significant slope when all data are combined in a weight of evidence determination. The slope appears to be much smaller at the highest

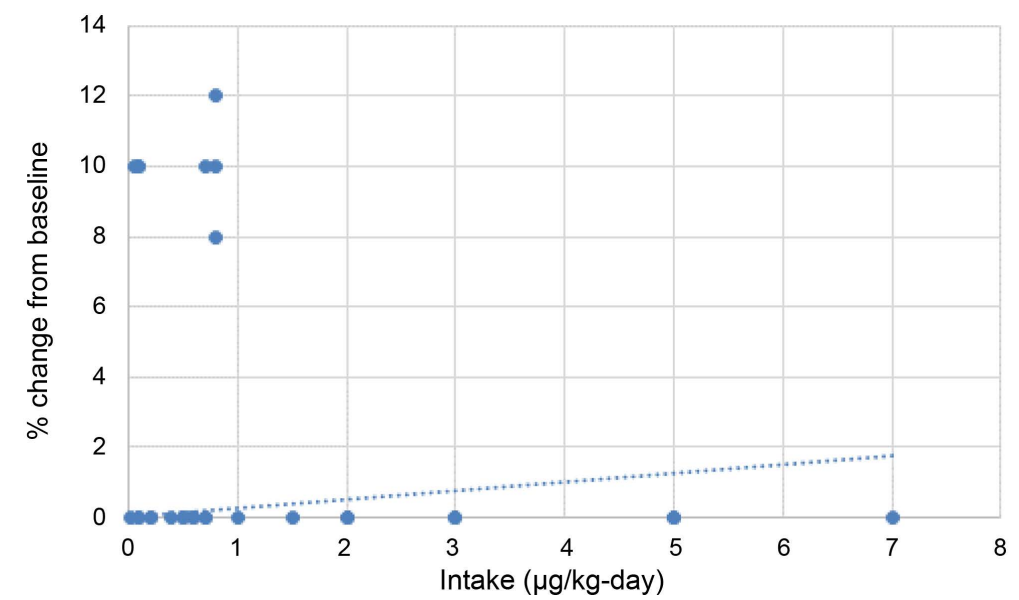

Figure 3. The regression analysis using all studies focused on hormone effects. No correction is made for the studies using urinary perchlorate as the "exposure measure". The mean slope in a linear regression is $0.3 \%$ per $\mu \mathrm{g} / \mathrm{kg}$-day. If half of the effects in the studies using urinary perchlorate are attributed to differences in $\lambda$ rather than differences in exposure, the mean slope reduces to $0.1 \%$ per $\mu \mathrm{g} / \mathrm{kg}$-day. Note that some studies lie over each other and therefore appear as a single data point in the figure. 
intakes, probably due to perchlorate uptake by the thyroid being a saturable process.

However, note also the shape of the exposure-response relationship for hormone effects. If the data are taken at face value, they appear to indicate effects appearing at the lowest exposures and then disappearing at approximately $1 \mu \mathrm{g} / \mathrm{kg}$-day and above. And if the finding of Greer et al. [14] of a small change in hormone levels at $500 \mu \mathrm{g} / \mathrm{kg}$-day is considered (bearing in mind that the EFSA considered this change not to be statistically significant), the possibility is raised of a u-shaped curve with effects at low exposures, no effects at intermediate exposures, followed by a slight increase again at the highest levels of exposure (approximately 4 orders of magnitude above environmentally relevant exposures). This raises the question of what could be accounting for this complex behavior of exposure-response for hormone effects by perchlorates.

To address this question, note that the large majority of epidemiological and clinical studies of the effects of exposure to perchlorates considered here have been based on the assumption that any elevation or decline in a metric of effect is due solely to the action of the incremental exposure to perchlorates. In the present analysis, these results are re-assessed through reflection on the potential impact of exposures to nitrates, thiocyanates and organohalogens as part of the mixture of compounds acting to produce the effects.

Turning first to the IUI results, the inclusion of a cumulative and aggregate risk methodology with exposures to the three goitrogen compounds expressed in units of $\mu \mathrm{g} / \mathrm{kg}$-day "compresses" the dynamic range of the exposures in the IUI graph, bringing the results of the different studies closer together in exposure-response relationship. If one takes the midpoints of results from studies 4, 11, 16 and 17, rather than relying entirely on the study of Greer et al. [14], the intake (PEC) associated with any given level of IUI increases by a factor of approximately 2.5 relative to the results obtained when cumulative and aggregate risk methods are applied to the Greer et al. [14] study, and by a factor of 200 to 300 relative to the results obtained when cumulative and aggregate risk are not applied to the Greer et al. [14] study. The former is a more reasonable difference in results than the latter, given that the studies used approximately the same dosing regime and examined healthy adults.

With respect to effects on serum thyroid hormone levels, the elevation of effects is found only in the studies 2, 9, 21, 24 and 26 that use urinary perchlorate as the metric of exposure, in contrast to the large majority of other studies that employ measures of environmental concentration (the basis of regulatory limits). As described in section 3, it is likely that a significant component of the differences in urinary concentration between individuals in the "exposure categories" of such studies are due to inter-subject variability in first order clearance rate constant rather than environmental concentration or intake rate.

The exception to the above paragraph is study 25 , where an elevation in TSH is found for exposures (measured as exposures, not as urinary perchlorate) slightly below $0.6 \mu \mathrm{g} / \mathrm{kg}$-day, corresponding to a perchlorate-only concentration of $18 \mu \mathrm{g} / \mathrm{L}$. However in that study, the effect was reversed after 24 hours, suggesting a rebound (in fact, 
over-compensation) by the homeostatic system. Still, the rebound was not evident in newborns with the highest levels of TSH, so this result stands out in contrast to the other studies. Again, it must be noted that there was no control for confounding by organohalogens, known to be potent in inducing effects on serum thyroid hormone levels and amongst the endocrine disruptors of concern in regulatory discussions.

The conclusion is that current epidemiological results do not provide evidence of effects induced by perchlorates apart from the IUI effects noted in Greer et al. [14]; nor do they provide evidence that the IUI effects induced at environmental levels of exposure are associated with down-stream adverse effects.

In addition, regulatory decisions include both the establishment and management of risk. This raises the question of whether effective risk management under cumulative and aggregate risk would focus on exposures to perchlorates, or might instead focus on the other compounds (nitrates, thiocyanates and organohalogens) known to induce the same IUI and hormone effects seen in Figure 2 and Figure 3. As shown in a previous study on this issue [18] use of cumulative and aggregate risk in reinterpreting the IUI effects through the additional lens of probabilistic risk assessment suggests that the large majority of individuals "at risk" from exposure to the three IUI-inducing compounds have that risk dominated by nitrates and thiocyanates. It is not possible at present to perform the same analysis for perchlorates and organohalogens, since the epidemiological studies shown in Figure 2 do not control for confounding by organohalogen exposures. It is recommended that these studies be reassessed to not only control for this potential confounding, but to provide separate estimates of the levels of hormone effects from exposure to perchlorates, nitrates, thiocyanates and organohalogens. Only when this is accomplished will there be a sound basis for risk management strategies addressing at least these four compounds through cumulative and aggregate risk.

\section{Acknowledgements}

This research has followed the principles of $\mathrm{REACH}^{1}$ (Registration, Evaluation, Authorisation and restriction of Chemicals) in the EU, which calls on the combined efforts of governments and industry to provide the data (industry) and analyses (government) needed for protection of public health from risk compounds in the environment and food. The authors therefore gratefully acknowledge the funding support provided by the USEPA (under the NEXGen program); American Water Works Association (water trade association policy group); and a consortium of industry representatives in the EU.

\section{References}

[1] Cote, I., Anastas, P., Birnbaum, L. Clark, R., Dix, D., Edwards, S., et al. (2012) Advancing the Next Generation of Health Risk Assessment. Environmental Health Perspectives, 120, 1499-1502. http://dx.doi.org/10.1289/ehp.1104870

[2] EFSA (2014) Modern Methodologies and Tools for Human Hazard Assessment of Chemicals. European Food Safety Authority. EFSA Journal, 12, 3638.

${ }^{1}$ http://ec.europa.eu/environment/chemicals/reach/reach_en.htm 
http://dx.doi.org/10.2903/j.efsa.2014.3638

[3] Sexton, K. (2012) Cumulative Risk Assessment: An Overview of Methodological Approaches for Evaluating Combined Health Effects from Exposure to Multiple Environmental Stressors. International Journal of Environmental Research and Public Health, 9, 370390. http://dx.doi.org/10.3390/ijerph9020370

[4] Ragasa, A., Oldenkamp, R., Preeker, N., Wernicke, J. and Schlink, U. (2011) Cumulative Risk Assessment of Chemical Exposures in Urban Environments. Environment International, 37, 872-881. http://dx.doi.org/10.1016/j.envint.2011.02.015

[5] USEPA (2003) Framework for Cumulative Risk Assessment. EPA/600/P-02/001F, US Environmental Protection Agency, Office of Research and Development, National Center for Environmental Assessment, Washington Office, Washington DC.

[6] Meek, M., Boobis, A., Crofton, K., Heinemeyer, G., Raaij, M. and Vickers, C. (2011) Risk Assessment of Combined Exposure to Multiple Chemicals: A WHO/IPCS Framework. Regulatory Toxicology and Pharmacology, 60, S1-S14.

http://dx.doi.org/10.1016/j.yrtph.2011.03.010

[7] EFSA (2013) International Frameworks Dealing with Human Risk Assessment of Combined Exposure to Multiple Chemicals. European Food Safety Authority. EFSA Journal, 11, 3313. http://dx.doi.org/10.2903/j.efsa.2013.3313

[8] Evans, R., Scholze, M. and Kortenkamp, A. (2015) Examining the Feasibility of Mixture Risk Assessment: A Case Study Using a Tiered Approach with Data of 67 Pesticides from the Joint FAO/WHO Meeting on Pesticide Residues (JMPR). Food and Chemical Toxicology, 84, 260-269. http://dx.doi.org/10.1016/j.fct.2015.08.015

[9] Evans, R., Martin, O., Faust, M. and Kortenkamp, A. (2015) Should the Scope of Human Mixture Risk Assessment Span Legislative/Regulatory Silos for Chemicals? Science of the Total Environment, 543, 757-764. http://dx.doi.org/10.1016/j.scitotenv.2015.10.162

[10] ICRP (2007) The 2007 Recommendations of the International Commission on Radiological Protection. ICRP Publication 103, International Commission on Radiological Protection, Annals of the ICRP 37 (2-4).

[11] USEPA (2010) Recommended Toxicity Equivalence Factors (TEFs) for Human Health Risk Assessments of 2,3,7,8-Tetrachlorodibenzo-P-Dioxin and Dioxin-Like Compounds. EPA/ 100/R-10/005, US Environmental Protection Agency.

[12] EFSA (2014) Scientific Opinion on the Risks for Human and Animal Health Related to the Presence of Modified Forms of Certain Mycotoxins in Food and Feed. EFSA CONTAM Panel (EFSA Panel on Contaminants in the Food Chain) European Food Safety Authority. EFSA Journal, 12, 3916. http://dx.doi.org/10.2903/j.efsa.2014.3916

[13] EFSA (2016) Scientific Opinion on the Appropriateness to Set a Group Health-Based Guidance Value for Zearalenone and Its Modified Forms. EFSA Panel on Contaminants in the Food Chain. European Food Safety Authority, EFSA Journal, 14, 4425.

[14] Greer, M., Goodman, G., Pleus, R. and Greer, S. (2002) Health Effects Assessment for Environmental Perchlorate Contamination: The Dose-Response for Inhibition of Thyroidal Radioiodine Uptake in Humans. Environmental Health Perspectives, 110, 927-937. http://dx.doi.org/10.1289/ehp.02110927

[15] Bruce, G., Corey, L., Mandel, J. and Pleus, R. (2013) Urinary Nitrate, Thiocyanate, and Perchlorate and Serum Thyroid Endpoints based on NHANES 2001 to 2002. Journal of Occupational and Environmental Medicine, 55, 52-58. http://dx.doi.org/10.1097/JOM.0b013e31826bb774

[16] Tonacchera, M., Pinchera, A., Dimida, A., Ferrarini, E., Agretti, P., Vitti, P., et al. (2004) 
Relative Potencies and Additivity of Perchlorate, Thiocyanate, Nitrate, and Iodide on the Inhibition of Radioactive Iodide Uptake by the Human Sodium Iodide Symporter. Thyroid, 14, 1012-1019. http://dx.doi.org/10.1089/thy.2004.14.1012

[17] Sanchez, C., Blount, B., Valentin-Blasini, L. and Krieger, R. (2007) Perchlorate, Thiocyanate, and Nitrate in Edible Cole Crops (Brassica sp.) Produced in the Lower Colorado River Region. Bulletin of Environmental Contamination and Toxicology, 79, 655-659.

http://dx.doi.org/10.1007/s00128-007-9292-6

[18] Crawford-Brown, D. (2015) Application of Probabilistic Risk Assessment in Establishing Perchlorate and Goitrogen Risk Mitigation Strategies. International Journal of Environmental Research and Public Health, 12, 10374-10390. http://dx.doi.org/10.3390/ijerph120910374

[19] Crawford-Brown, D. and Crawford-Brown, S. (2016) Regulatory Rationality and the Role of Cumulative Risk: A Case Study of Perchlorates and Related Compounds. Cambridge Centre for Climate Change Mitigation Research, Working Paper 13.

http://www.4cmr.group.cam.ac.uk/filecab/perchlorate-rationality

[20] USEPA (2012) Life Stage Consideration and Interpretation of Recent Epidemiological Evidence to Develop a Maximum Contaminant Level Goal for Perchlorate. US Environmental Protection Agency White Paper, Health and Ecological Criteria Division, Office of Science and Technology, Office of Water, Washington DC.

[21] EFSA (2014) Scientific Opinion on the Risks to Public Health Related to the Presence of Perchlorate in Food, in particular Fruits and Vegetables. EFSA Panel on Contaminants in the Food Chain. EFSA Journal, 12, 3869 (117 pp).

[22] SAB (2013) SAB Advice on Approaches to Derive a Maximum Contaminant Level Goal for Perchlorate. EPA-SAB-13-004, USEPA Science Advisory Board. http://www.yosemite.epa.gov/sab\%5CSABPRODUCT.NSF/86E44EE7F27EEC1A85257B7B 0060F364/\$File/EPA-SAB-13-004-unsigned2.pdf

[23] Kodavanti, P. and Curras-Collazo, C. (2010) Neuroendocrine Actions of Organohalogens: Thyroid Hormones, Arginine Vasopressin, and Neuroplasticity. Frontiers in Neuroendocrinology, 31, 479-496. http://dx.doi.org/10.1016/j.yfrne.2010.06.005

[24] Charnley, G. (2008) Perchlorate: Overview of Risks and Regulation. Food and Chemical Toxicology, 46, 2307-2315. http://dx.doi.org/10.1016/j.fct.2008.03.006

[25] Tarone, R., Lipworth, L. and McLaughlin, J. (2010) The Epidemiology of Environmental Perchlorate Exposure and Thyroid Function: A Comprehensive Review. Journal of Occupational and Environmental Medicine, 52, 653-660. http://dx.doi.org/10.1097/JOM.0b013e3181e31955

[26] Makey, C., McClean, M., Braverman, L., Pearce, E., He, X.-M., Sjödin, A., Weinberg, J. and Webster, T. (2016) Polybrominated Diphenyl Ether Exposure and Thyroid Function Tests in North American Adults. Environmental Health Perspectives, 124, 420-425.

[27] Amitai, Y., Winston, G., Sack, J., Wasser, J., Lewis, M., Blount, B., Valenti-Blasini, L., Fisher, N., Israeli, A. and Leventhal, A. (2007) Gestational Exposure to High Perchlorate Concentrations in Drinking Water and Neonatal Thyroxine Levels. Thyroid, 17, 843-885. http://dx.doi.org/10.1089/thy.2006.0336

[28] Blount, B., Pirkle, J., Osterloh, J., Valentin-Blasini, L. and Caldwell, K. (2006) Urinary Perchlorate and Thyroid Hormone Levels in Adolescent and Adult Men and Women living in the US. Environmental Health Perspectives, 114, 1865-1871.

[29] Braverman, L., He, X., Pino, S., Cross, M., Magnani, B., Lamm, S., Kruse, M., Engel, A., Crump, K. and Gibbs, J. (2005) The Effect of Perchlorate, Thiocyanate, and Nitrate on 
Thyroid Function in Workers Exposed to Perchlorate Long-Term. Journal of Clinical Endocrinology and Metabolism, 90, 700-706. http://dx.doi.org/10.1210/jc.2004-1821

[30] Braverman, L., Pearce, E., He, X., Pino, S., Seeley, M., Beck, B., Magnani, B., Blount, B. and Firek A. (2006) Effects of Six Months of Daily Low-Dose Perchlorate Exposure on Thyroid Function in Healthy Volunteers. Journal of Clinical Endocrinology and Metabolism, 91, 2721-2724. http://dx.doi.org/10.1210/jc.2006-0184

[31] Brechner, R., Parkhurst, G., Humble, W., Brown, M. and Herman W. (2000) Ammonium Perchlorate Contamination of Colorado River Drinking Water Is Associated with Abnormal Thyroid Function in Newborns in Arizona. Journal of Occupational and Environmental Medicine, 42, 777-782. http://dx.doi.org/10.1097/00043764-200008000-00002

[32] Buffler, P., Kelsh, M., Lau, E., Edinboro, C., Barnard, J., Rutherford, G., Daaboul, J., Palmer, L. and Lorey, F. (2006) Thyroid Function and Perchlorate in Drinking Water: An Evaluation among California Newborns, 1998. Environmental Health Perspectives, 114, 798-804. http://dx.doi.org/10.1289/ehp.8176

[33] Cao, Y., Blount, B., Valentin-Blasini, L., Bernbaum, J., Phillips, T. and Rogan, W. (2010.) Goitrogenic Anions, Thyroid Stimulating Hormone, and Thyroid Hormone in Infants. Environmental Health Perspectives, 118, 1332-1337. http://dx.doi.org/10.1289/ehp.0901736

[34] Chang, S., Crothers, C., Lai, S. and Lamm, S. (2003) Pediatric Neurobehavioral Diseases in Nevada Counties with Respect to Perchlorate in Drinking Water: An Ecological Inquiry. Birth Defects Research Part A: Clinical and Molecular Teratology, 67, 886-892. http://dx.doi.org/10.1002/bdra.10089

[35] Charatcharoenwitthaya, N., Ongphiphadhanakul, B., Pearce, E., Somprasit, C., Chanthasenanont, A., He, X., Chailurkit, L. and Braverman, L. (2014) The Association between Perchlorate and Thiocyanate Exposure and Thyroid Function in First-Trimester Pregnant Thai Women. Journal of Clinical Endocrinology and Metabolism, 99, 2365-2371. http://dx.doi.org/10.1210/jc.2013-3986

[36] Crump, C., Michaud, P., Tellez, R., Reyes, C., Gonzalez, G., Montgomery, E., Crump, K., Lobo, G., Becerra, C. and Gibbs, J. (2000) Does Perchlorate in Drinking Water Affect Thyroid Function in Newborns or School-Age Children? Journal of Occupational and Environmental Medicine, 42, 603-612. http://dx.doi.org/10.1097/00043764-200006000-00009

[37] Kelsh, M., Buffler, P., Daaboul, J., Rutherford, G., Lau, E., Barnard, J., Exuzides, A., Madl, A., Palmer, L. and Lorey, F. (2003) Primary Congenital Hypothyroidism, Newborn Thyroid Function, and Environmental Perchlorate Exposure among Residents of a Southern California Community. Journal of Occupational and Environmental Medicine, 45, 1116-1127. http://dx.doi.org/10.1097/01.jom.0000091683.25325.55

[38] Lamm, S., Braverman, L., Li, F., Richman, K., Pino, S. and Howearth, G. (1999) Thyroid Health Status of Ammonium Perchlorate Workers: A Cross-Sectional Occupational Health Study. Journal of Occupational and Environmental Medicine, 41, 248-260. http://dx.doi.org/10.1097/00043764-199904000-00006

[39] Lamm, S. and Doemland, M. (1999) Has Perchlorate in Drinking Water Increased the Rate of Congenital Hypothyroidism? Journal of Occupational and Environmental Medicine, 41, 409-411. http://dx.doi.org/10.1097/00043764-199905000-00011

[40] Lamm, S. (2003) Perchlorate Exposure Does Not Explain Differences in Neonatal Thyroid Function between Yuma and Flagstaff. Journal of Occupational and Environmental Medicine, 45, 1131-1132. http://dx.doi.org/10.1097/01.jom.0000094991.31330.d3

[41] Lawrence, J., Lamm, S., Pino, S., Richman, K. and Braverman, L. (2000) The Effect of ShortTerm Low Dose Perchlorate on Various Aspects of Thyroid Function. Thyroid, 10, 659663. http://dx.doi.org/10.1089/10507250050137734 
[42] Lawrence, J., Lamm, S. and Braverman, L.E. (2001) Low Dose Perchlorate (3 mg Daily) and Thyroid Function. Thyroid, 11, 295. http://dx.doi.org/10.1089/105072501750159796

[43] Leung, A., Braverman, L., He, X., Schuller, K., Roussilhes, A., Jahreis, K. and Pearce, E (2012) Environmental Perchlorate and Thiocyanate Exposures and Infant Serum Thyroid Function. Thyroid, 22, 938-943. http://dx.doi.org/10.1089/thy.2012.0058

[44] Li, Z., Li, F.X., Byrd, D., Deyhle, G.M., Sesser, D.E., Skeels, M.R. and Lamm, S.H. (2000) Neonatal Thyroxine Level and Perchlorate in Drinking Water. Journal of Occupational and Environmental Medicine, 42, 200-205. http://dx.doi.org/10.1097/00043764-200002000-00020

[45] Li, F.X., Squartsoff, L. and Lamm, S. (2001) Prevalence of Thyroid Diseases in Nevada Counties with Respect to Perchlorate in Drinking Water. Journal of Occupational and Environmental Medicine, 43, 630-634. http://dx.doi.org/10.1097/00043764-200107000-00010

[46] Mendez Jr., W. and Eftim, S.E. (2012) Biomarkers of Perchlorate Exposure Are Correlated with Circulating Thyroid Hormone Levels in the 2007-2008 NHANES. Environmental Research, 118, 137-144. http://dx.doi.org/10.1016/j.envres.2012.05.010

[47] Pearce, E., Lazarus, J., Smyth, P., He, X., Dall'Amico, D., Parkes, A., Burns, R., Smith, D., Maina, A., Bestwick, J., Jooman, M., Leung, A. and Braverman, L. (2010) Perchlorate and Thiocyanate Exposure and Thyroid Function in First-Trimester Pregnant Women. Journal of Clinical Endocrinology and Metabolism, 95, 3207-3215. http://dx.doi.org/10.1210/jc.2010-0014

[48] Pearce, E., Spencer, C., Mestman, J., Lee,R., Bergoglio, L., Mereshian, P., He, X., Leung, A. and Braverman, L. (2011) Effect of Environmental Perchlorate on Thyroid Function in Pregnant Women from Cordoba, Argentina, and Los Angeles, California. Endocrinology Practice, 17, 412-417. http://dx.doi.org/10.4158/EP10293.OR

[49] Steinmaus, C., Miller, M. and Howd, R. (2007) Impact of Smoking and Thiocyanate on Perchlorate and Thyroid Hormone Associations in the 2001-2002 National Health and Nutrition Examination Survey. Environmental Health Perspectives, 115, 1333-1338. http://dx.doi.org/10.1289/ehp.10300

[50] Steinmaus, C., Miller, M.D. and Smith, A.H. (2010) Perchlorate in Drinking Water during Pregnancy and Neonatal Thyroid Hormone Levels in California. Journal of Occupational and Environmental Medicine, 52, 1217-1524. http://dx.doi.org/10.1097/JOM.0b013e3181fd6fa7

[51] Steinmaus, C., Miller, M., Cushing, L., Blount, B. and Smith, A. (2013) Combined Effects of Perchlorate, Thiocyanate, and Iodine on Thyroid Function in the National Health and Nutrition Examination Survey 2007-2008. Environmental Research, 123, 17-24. http://dx.doi.org/10.1016/j.envres.2013.01.005

[52] Suh, M., Abraham, L., Hixon, J. and Proctor, D. (2013) The Effects of Perchlorate, Nitrate, and Thiocyanate on Free Thyroxine for Potentially Sensitive Subpopulations of the 20012002 and 2007-2008 National Health and Nutrition Examination Surveys. Journal of Exposure Science and Environmental Epidemiology, 24, 579-587. http://dx.doi.org/10.1038/jes.2013.67

[53] Tellez, R., Chacon, P., Abarca, C., Blount, B., Van Landingham, C., Crump,K. and Gibbs, J. (2005) Long-Term Environmental Exposure to Perchlorate through Drinking Water and Thyroid Function during Pregnancy and the Neonatal Period. Thyroid, 15, 963-975. http://dx.doi.org/10.1089/thy.2005.15.963

[54] Leung, A., Katz, P., He, X, Feig, D., Pearce, E. and Braverman, L. (2014) Urinary Perchlorate and Thiocyanate Concentrations in Pregnant Women from Toronto, Canada. Thyroid, 24, 175-176. http://dx.doi.org/10.1089/thy.2013.0228 
[55] English, P., Blount, B., Wong, M., Copan, L., Olmedo, L., Patton, S., Haas, R., Atencio, R., $\mathrm{Xu}$, J. and Valentin-Blasini, L. (2011) Direct Measurement of Perchlorate Exposure Biomarkers in a Highly Exposed Population: A Pilot Study. PLoS ONE, 6, e17015.

http://dx.doi.org/10.1371/journal.pone.0017015

[56] Lau, F., deCastro, B., Mills-Herring, L., Lin, T., Valentin-Blasini, L., Udeni Alwis, K. and Blount, B. (2012) Urinary Perchlorate as a Measure of Dietary and Drinking Water Exposure in a Representative Sample of the United States Population 2001-2008. Journal of EXposure Science and Environmental Epidemiology, 23, 207-214.

http://dx.doi.org/10.1038/jes.2012.108

[57] Pearce, N., Checkoway, H. and Kriebel, D. (2007) Bias in Occupational Epidemiology Studies. Journal of Occupational and Environmental Medicine, 64, 562-568.

http://dx.doi.org/10.1136/oem.2006.026690

Submit or recommend next manuscript to SCIRP and we will provide best service for you:

Accepting pre-submission inquiries through Email, Facebook, LinkedIn, Twitter, etc.

A wide selection of journals (inclusive of 9 subjects, more than 200 journals)

Providing 24-hour high-quality service

User-friendly online submission system

Fair and swift peer-review system

Efficient typesetting and proofreading procedure

Display of the result of downloads and visits, as well as the number of cited articles

Maximum dissemination of your research work

Submit your manuscript at: http://papersubmission.scirp.org/

Or contact jep@scirp.org 